\title{
Dependent and Independent Reasons
}

\author{
ROBERT J. YANAL Wayne State University
}

Key Terms: Linked premises; convergent premises; induction; deduction.

Abstract: How are dependent (or linked) premises to be distinguished from independent (or convergent) premises? Deductive validity, sometimes proposed as a necessary condition for dependence, cannot be, for the premises of both inductive and deductive but invalid arguments can be dependent. The question is really this: When do multiple premises for a certain conclusion form one argument for that conclusion and when do they form multiple arguments? Answer: Premises are dependent when the evidence they offer for their conclusion is more than the ordinary sum of their probabilities. Ordinary sums are defined in the paper.

Teaching the structure of arguments using argument diagrams has become a commonplace in informal logic. The main graphic device of these diagrams is an arrow running from premise (reason, evidence) to conclusion. The simplest structure any argument can have is this:

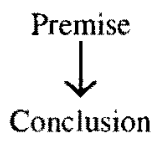

The pedagogical value of diagrams shines when students are confronted with more complex pieces of reasoning-reasons for reasons, subconclusions, main conclusions, and so on. It is possible to diagram arguments of some complexity:

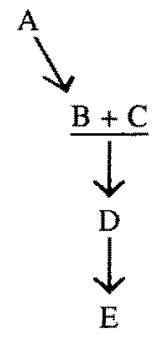

As can be seen, argument diagrams allow students to draw more distinctions than the older, traditional exercises that simply asked them to underline a conclusion. Argument diagrams can differentiate main from subconclusions; they can deal with multiple premises; and can display what is being offered as a reason for what else, and what has had no reason offered for it at all.

As it happens, textbooks that use argument diagrams draw a distinction between two kinds of relations among premises, which we will call "dependent" and "independent" reasons." The distinction graphically is this:
Dependent

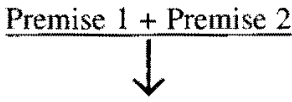

Conclusion
Independent

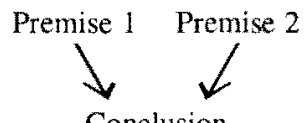

Conclusion
This pair draws the contrast we are after:
A. $<$ Al $>$ Either she's in the kitchen or she's in the study. $\angle A 2>$ She's not in the kitchen. So $<A 3>$ she's in the study.
B. $\langle$ B $>$ Smoking in this building is pun- ishable by a fine. Besides, $\angle \mathrm{B} 2>$ smoking increases your risk of heart attack. There- fore, $<$ B3 $>$ it's against your self-interest to light up here.

It is just such cases as A and B that lead us to think there is a real distinction to be drawn, though we must wait a bit to see exactly what the distinction amounts to and why it is important to teach students to draw it.

The terms "dependent" and "independent" imply a raw way to understand the distinction. Dependent premises depend on each other to produce their conclusion; 
independent premises do not. More helpfully we can say that the premises of A fill in each other's logical gaps, or are in the same line of thought, or present one unified piece of reasoning; and that the premises of B do not fill in each other's logical gaps, or are not in the same line of thought, or present separate pieces of reasoning. ${ }^{2}$ These are acceptable descriptions of the distinction-and may better impart the distinction to introductory students, I should think, than the differentia I will shortly propose which require some familiarity with manipulating probabilities.

While it may be pedagogically useful to speak of "filling in logical gaps" or "being in separate lines of thought," we should seek a deeper explanation of the conceptual difference between the relations among premises in arguments A and B. I want to ask: What does the dependentindependent distinction distinguish? What are dependent and independent reasons?

Everyone who uses the diagram method would, I think, agree that reasons $\mathrm{A} 1$ and $\mathrm{A} 2$ are dependent, reasons $\mathrm{B} 1$ and $\mathrm{B} 2$ independent:

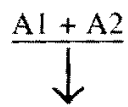

A3

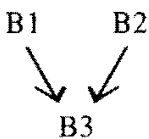

B3
Yet what, intuitively, is going on? Well, argument A is deductively valid, hence deductive. Argument $B$ is non-deductive. Are dependent reasons coextensive with (and only with) the premises of deductively valid arguments?

No. Reasons in other kinds of arguments can also be dependent. The premises of simple inductive generalizations and most deductive fallacies are dependent not independent:

The crows induction. Crow \#1 is observed to be black. Crow \#2 is also observed to be black. ... Crow \#100 is observed to be black. So, all crows are black.

The lightning fallacy. If lightning strikes the barn, then the barn burns down. And the barn is burning down. So lightning struck it.

If the reasons in these two arguments are dependent-and I think they are, though we will have to wait a bit for an explanation of this-then deductive validity cannot be the criterion of dependent reasons.

If, besides deductively valid arguments, at least some inductions and even some deductive fallacies have dependent premises, we must look for an explanation of dependence that does not collapse into deductive validity. We'll look at several authors who try to explicate the dependence of the premises on one another.

Irving Copi writes that in arguments with dependent reasons "neither of the two premises supports the conclusion independently." Copi's idea seems to be that both premises in argument $\mathrm{A}$ offer support to the conclusion, but only jointly; and that's why $\mathrm{A} 1$ and $\mathrm{A} 2$ are dependent. In contrast, B1 and B2 considered separately do each support their conclusion; and that's why they are independent.

However, Copi discounts the fact that in most deductive arguments at least one premise provides some support for its conclusion. Regarding example A, we wouldn't count the argument, "She's not in the kitchen, so she's in the study" as a complete non sequitur. If I ask, "Where is she?" and you answer, "Well, she's not in the kitchen," I may not have much to go on, but I'll have to count your information as relevant. But this means that Copi's criterion shows A to have, wrongly, independent premises, for at least one of its premises supports the conclusion independently.

In addition, the no-independentsupport test doesn't seem to work on simple inductions. Consider the crows induction. Each premise considered separately offers some support for the conclusion, which would entail that each premise is independent. Yet we think the premises of 
enumerative inductions to be dependent. We think in the crows induction we have one argument, not one hundred.

Stephen Thomas writes that in arguments with independent premises "each separate reason still would support the conclusion just as well even if the other (separate, independent) reason(s) were false ...."4 This is true. In the independently premised argument, $P I, P 2$, therefore $C, \mathrm{P} 2$ supports the conclusion "just as well" even supposing P1 false. The degree of support, $d$, offered to $\mathrm{C}$ by $\mathrm{P} 1$ is neither raised nor lowered by the falsehood of $\mathrm{P} 2$. (It is also neither raised nor lowered by the truth of P2.)

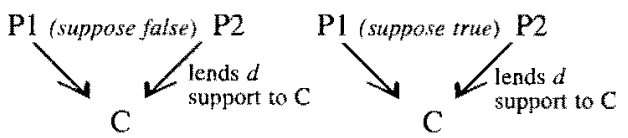

However, the converse of Thomas's proposal is that in arguments whose reasons are dependent, the falsehood of one dependent reason would weaken the support of the whole argument. But consider a deductively valid argument which has a false premise. Those premises still support their conclusion "just as well," for the argument is still deductively valid:

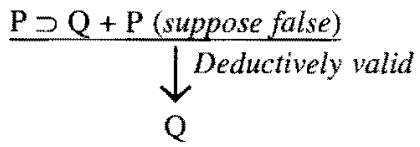

Of course, there is a clear sense in which the true premises of deductively valid arguments support their conclusions better than false premises of deductively valid arguments. In a variation on Thomas's proposal, Trudy Govier tries to capture this sense and escape the objection just made to Thomas. She writes, "If either premise [in an argument with dependent premises] is false or unacceptable, the whole argument will be flawed, since each premise needs the other to support the conclusion." ${ }^{\text {s }}$ However, Govier's proposal requires that we already know that we have one "whole argument" and not two.
Consider again argument $\mathrm{A}$, the disjunctive syllogism stated earlier. Suppose I wrongly think $A$ 's premises to be independent. Suppose, that is, that I think that there are two whole arguments. In addition, suppose that she is in the kitchen, that is, that $\mathrm{A} 2$ is false:

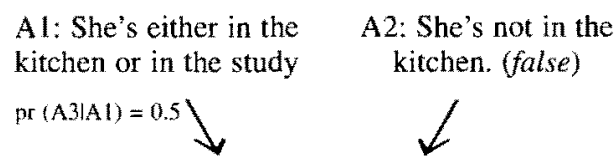

A3: She's in the study.

The notations say this: Given only that she's either in the kitchen or in the study, then without any further background information, there is a 0.5 probability that she's in the study. However, the premise I think independent, A2, is false. Still, I can be quite confident that premise A1 still renders conclusion A3 0.5 probable. In other words, it appears that the argument flawed by falsehood, namely $\mathrm{A} 2 \rightarrow \mathrm{A} 3$, does not impinge on the argument $\mathrm{A} 1 \rightarrow \mathrm{A} 3$. But Govier's criterion should not thus support me when I take A1 and A2 to be independent.

What, then, is the difference between dependent and independent reasons? I suggest that it is this: Dependent reasons form one argument; independent reasons form multiple arguments. Indeed, the very notation of independence suggests this-one arrow denoting one argument, two arrows denoting two:

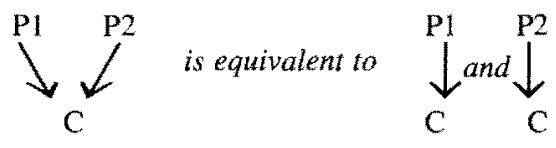

The "and" means no more than "is stated concurrently with." Of course, the two inferences, $\mathrm{P} 1 \rightarrow \mathrm{C}$ and $\mathrm{P} 2 \rightarrow \mathrm{C}$, could each be treated as enthymemes, resulting in two deductive arguments when the missing premise is supplied. If dependent reasons form a single argument and independent reasons form multiple arguments, then we need a way of individuating 
arguments. We want a conception of oneness or wholeness of arguments that will show, given that we are in the presence of some number of arguments, when we have reasons arrayed in one argument and when we have reasons that make more than one argument.

This brings us to the reason why the independent-dependent distinction is worth drawing and teaching students to draw. In arguments with independent reasons, it is entirely possible for one reason to support the conclusion weakly and another to support it strongly-or, in other words, it is entirely possible to have two arguments for the same conclusion, one weak, the other strong.

C. $\langle\mathrm{Cl}\rangle$ The fortune teller using Tarot cards predicts that the stock market will go up. $\langle\mathrm{C} 2\rangle$ The economist using the third quarter earnings reports predicts that the stock market will go up. Therefore, $\langle\mathrm{C} 3\rangle$ the stock market will go up.

$\mathrm{C}$ gives us two arguments from authority. One uses an unreliable authority, the other a more reliable authority. (It is an exercise left to the reader which is which.) One must be labeled weaker, the other stronger. This would be entirely lost if $\mathrm{C} 1$ and $\mathrm{C} 2$ were somehow blended together as if dependent. There is no sense in saying that a weak reason added to a strong reason average out into one moderate argument. On the other hand, when reasons are dependent, there is no sense in evaluating them separately. Two dependent reasons produce their conclusions strongly or weakly, but they do it as if they were one reason stated in two parts.

A criterion I proposed elsewhere ${ }^{6}$ is this: The probability of the conclusion of an argument with independent premises is the ordinary sum of the probability of each premise. The probability of the conclusion of an argument with dependent premises is not the ordinary sum of the probability of each premise. I also like to put it this way: The probability of conclusions from arguments whose premises are dependent jumps beyond the ordinary sum of these premises. I would like to expand my proposal, and extend it to inductions and certain deductive fallacies.

What is the ordinary sum of evidence? Consider the argument, $P 1, P 2$, therefore $C$. Suppose $P 1$ in itself lends 0.3 probability to C. Suppose P2 in itself lends 0.4 probability to $\mathrm{C}$. Clearly we know $\mathrm{C}$ with some probability greater than 0.4. But what? We can think of the ordinary sum of probabilities this way. The probability of $\mathrm{C}$ given $P 1$ is 0.3 . Take 0.3 from 1.0, leaving 0.7. Call 0.7 "what is unknown." When P2 is brought in, we know 0.4 of 0.7 , or $0.4 x$ 0.7 , which is 0.28 more than we knew before. In sum we know the conclusion $\mathrm{C}$ with 0.58 probability $(=0.3+0.28)$.

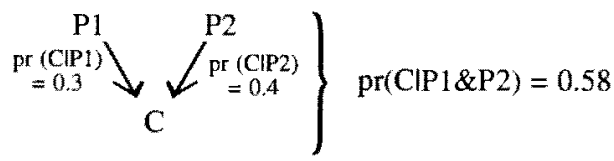

It works quite differently with argument $A$. We know that its premises confer a probability of 1.0 -certainty-on their conclusion, since the argument is deductively valid. Were we to sum them ordinarily, and assigning arbitrarily the probability of 0.1 to the conclusion on the minor premise, we would get something like this:

$$
\left.\begin{array}{l}
\text { A1: Either A2: Not in } \\
\text { in the kitchen the kitchen } \\
\text { or in the study } \\
\begin{array}{l}
\mathrm{pr}(\mathrm{A} 3 \mathrm{~A} \mid \mathrm{A}) \\
=0.5
\end{array} \\
\mathrm{pr}(\mathrm{A} \text { 3|A2) } \\
=0.1
\end{array}\right\} \operatorname{pr}(\mathrm{A} 3 \mid \mathrm{A} 1 \& \mathrm{~A} 2)=0.55
$$

A3: In the study.

But this is incorrect: We know that the premises of A confer 1.0 probability on their conclusion. So the premises must sum nonordinarily. ${ }^{\text {? }}$

Writers on the dependent-independent difference seem to ignore inductions, which are usually dependent arguments. Reconsider the earlier example of one hundred observations made of black crows, from which is induced the conclusion that all crows are black. The dependence in the 
crows induction relies on an intuitive claim about the accumulation of evidence. To treat its premises, that is, each individual observation that a certain crow is black, as independent, one must imagine oneself treating each crow observation as giving a miniscule probability for the conclusion that all crows are black, then summing all one hundred of them ordinarily. However, one hundred independent observations of one hundred black crows gives greater than the ordinary sum of one hundred miniscule probabilities, and so its premises are dependent:

Crow \#1 is black + Crow $\# 2$ is black + $\frac{\ldots+\text { Crow \#100 is black }}{\downarrow}$

All crows are black.

Here's why. At some point in the accumulation of evidence, evidence acquires strength beyond the sum of its parts. Suppose we take the textbook example of drawing ten black marbles drawn at random out of a jar of one hundred marbles. We then conclude that all marbles in the jar are black. Summed ordinarily, taking each premise independently, the premises confer approximately 0.1 probability on their conclusion (actually it's about 0.0956 using my method of ordinary sums):

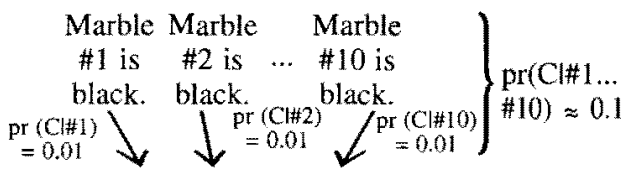

C: All 100 marbles in the jar are black.

But I think we have better reason to believe the conclusion than this. I think it is clear that we have better than a $10 \%$ chance that all the marbles in the urn are black, given a random drawing of ten balls each of which is black. So the premises in inductive generalizations sum nonordinarily, and are thus dependent.

Some deductively invalid arguments, such as the lightning argument earlier, also have dependent premises. This is hard to see if it is thought that a fallacy is an argument whose premises give no support for their conclusion. Deductive fallacies fail to entail their conclusion deductively, though their premises often offer some inductive support. In the lightning fallacy earlier, we have an instance of the deductive fallacy of affirming the consequent. Suppose we begin, wrongly, to consider the argument as having independent premises, and, wrongly, to sum the probabilities conferred on the conclusion ordinarily:

$$
\begin{aligned}
& \text { P1: If lightning } \quad \mathrm{P} 2 \text { : The barn } \\
& \text { strikes it, the barn burns down. } \\
& \text { burns down. } \begin{array}{c}
\text { pr (ClP2) }=0 \\
\operatorname{pr}(\mathrm{C} \mid \mathrm{P} 1) \approx 0 \\
\text { a virtual non } \\
\text { sequitur. }
\end{array} \\
& \text { C: Lightning struck the barn. }
\end{aligned}
$$

This is wrong because the premises jointly give greater reason to believe the conclusion then their ordinary sum of approximately zero. For each premise, separately, offers virtually no support for its conclusion; and the ordinary sum of the probability the premises offer to their conclusion is approximately zero. But looking at the argument as a whole, even though the premises don't deductively entail the conclusion, they still offer slight support for it, certainly greater than virtually no support. After all, if $P$ is a sufficient reason for $Q$, and $Q$ obtains, don't we have some reason to believe that $P$ obtained as well? And that is what makes the reasons in deductive fallacies dependent.

\section{If lightning strikes the barn, $\quad+\quad$ The barn then it burns down. burns down.

$\downarrow^{\text {slight, not nil, suppor }}$ Lightning struck it.}

There is thus one unitary argument, and although logically weak, it is stronger than the sum of the two premises considered independently.

Hard cases abound in informal logic, and sometimes clearly establishing some reasons as dependent or as independent is difficult. What about the premises of this argument? 
D. $<$ D1 $>$ She was seen heading in the general direction of the library. $\angle D 2>$ Her exams are coming up. So $\langle$ D 3$\rangle$ she's at the library.

Argument $\mathrm{D}$ is non-deductive. Do its premises join dependently? Or are there two arguments going on? Here, I think, is another case which the Thomas-Govier criterion doesn't help. Assume we discover D1 to be false. Until we know if we are dealing with one argument and not two, we won't know whether the falsehood of D1 infects "the" argument "as a whole," If D1 and D2 form independent arguments, the inference D2 $\rightarrow$ D3 will remain unaffected by the flawed (unsound) argument, D $1 \rightarrow$ D3.

My criterion, while initially stated in terms of credibly assignable probabilities to conclusions given certain premises, can provide a way of thinking of reasons that is not tied to adding definite numbers. Think of it this way. Someone begins by citing D1 as a reason to believe D3, and clearly D1 provides some evidence for D3. But then he continues, and offers D2 as additional evidence for D3. Obviously we now have more evidence, and hence more reason to believe D3. The critical question is this: Does the overall evidence given in argument D "jump" when D2 is brought in? Then D1 and D2 are dependent reasons. Or are we left only with gradually increasing evidence? Then D1 and D2 are independent reasons. Will our credence in D3 be reduced by more than the evidence brought in by D1 if D1, say, is discovered false? Or will our credence be reduced by just that evidence? If the former, the reasons are dependent; if the latter, they are independent. Fleshing out what I take to be our basic intuition about the dependentindependent reasons distinction has not provided a clear litmus test for any argument, but only a line of questioning to be undertaken.

There are odd truth functional constructions in which either one reason deductively entails its conclusion and the other does not; or each reason deductively entails its conclusion. Consider these:

E. $\langle$ El $>$ If it is daytime, it is daytime. $<\mathrm{E} 2>$ It is daytime. So $<\mathrm{E} 3\rangle$ it is daytime.

F. $\langle\mathrm{Fl}\rangle$ It is raining. $\langle\mathrm{F} 2\rangle$ The wind is blowing. So, $\langle\mathrm{F} 3\rangle$ either Rembrandt painted The Polish Rider or Rembrandt did not paint The Polish Rider.

If we treat $\mathrm{E}$ as an argument, then we will say that the inference $(\mathrm{E} 1+\mathrm{E} 2) \rightarrow \mathrm{E} 3$ is deductively valid (or E3 is 1.0 probable given E1 and E2). But the inference from E2 to E3 is also deductively valid. So: Are the premises E1 and E2 dependent or independent? Since 1.0 probability can hardly be improved upon, either the ordinary or the nonordinary sum of $\mathrm{E} 1$ and $\mathrm{E} 2$ yields $\mathrm{C}$ with 1.0 probability. So my test can't give an answer one way or the other (or worse, it says that the premises of $E$ are both dependent and independent).

$F 1$ and F2 present a similar situation. If $\mathrm{F}$ is an argument, $\mathrm{F} 3$ is deductively entailed by either F1 or F2 (or anything else for that matter), for F3 is a tautology, hence a logical truth, hence cannot figure as a false conclusion. Again my iest can't apply, for my test invites consideration of how likely reasons make conclusions and how this likelihood comes about (ordinarily or nonordinarily); and the premises of $F$ don't confer any likelihood on F3 at all, though F3 is still as likely true as one can have it. Some might think this a death blow to my test. However to say this is to put forth intuitions that the premises of arguments $\mathrm{E}$ and $\mathrm{F}$ are clearly dependent. And I don't think we have such intuitions.

Indeed, it is not clear to me that $\mathrm{E}$ and $F$ are arguments. Of course, both $E$ and $F$ are instances of truth functionally valid forms. $E$ is of the form,

$$
\mathrm{P} \supset \mathrm{Q}, \mathrm{P} \text {, therefore } \mathrm{Q}
$$

$F$ is of the form,

$\mathrm{P}, \mathrm{Q}$, therefore $\mathrm{R}$ or $\rightarrow \mathrm{R}$. 
My own view is that not every instantiation of these forms counts as an argument, if by "argument" we mean "the giving of evidence for something or the presenting of reasons to believe something." That, after all, is the informal logic or ordinary language sense of argument; and the dependentindependent distinction is a feature of informal logic. Neither $\mathrm{E}$ nor $\mathrm{F}$ is an argument in this sense. $E$ sounds like someone repeating himself, not giving reasons to believe that some statement is true. $F$ is incomprehensible to those not initiated into the mysteries of truth tables; and even those conversant with valid truth functional argument forms agree that $\mathrm{F}$ is a kind of anomaly or paradox that unfortunately but unavoidably comes with the territory. I say "unfortunately" for we cannot, speaking ordinarily, deduce F's conclusion from its premises, where "deduce" is used in its central sense of "drawing out what is contained therein."

Now, if someone wishes to say that any instantiation of a deductively valid schema, even $\mathrm{E}$ and $\mathrm{F}$, counts as an argument; and that the premises of every argument that has more than one premise must be either dependently or independently related; then he will seek a different account of the distinction than the one I have offered or will seek to supplement it in different ways. Perhaps he will say that every instance of a deductively valid form will have dependent premises (if it has at least two premises). But why would this point be pressed? Why exactly do the premises in any instance of a deductively valid form relate dependently? And, especially, how does whatever reason put forth to support this apply to deductively invalid arguments and to inductions? Or perhaps he will say that we must look to the speaker's intentions to see whether premises are dependent or independent. This won't do by itself, for we must have an understanding of dependence and independence before we can have an understanding of intending premises to be dependent (or independent); and we must also have an argument for the view that intending premises to be dependent makes them so.

I have been asked how my criterion applies to such peculiar prose as:

G. $\langle\mathrm{G} 1\rangle$ Sugar is sweet. $\langle\mathrm{G} 2\rangle$ The moon is a satellite of the earth. Therefore $\langle\mathrm{G} 3\rangle$ today is Tuesday.

If we wish to treat this as an argument and apply my criterion, we will say that the probability of G3 given $\mathrm{G} 1$ or G2, summed ordinarily or nonordinarily, is zero. Hence G1 and G2 cannot be said, by my criterion, to be dependent or independent. Those who say that either $\langle\mathrm{G} 1\rangle$ and $\langle\mathrm{G} 2\rangle$ are dependent premises or are independent premises must defend the presupposition that $\mathrm{G}$ is an argument at all. And on what grounds would this be defended? Because it contains "therefore"? "Therefore" signals an argument only when the word is used in the sense of "is offered as a reason for." So we must then ask: Is there an inference to the best explanation of the intentions of the maker of $\mathrm{G}$ such that $\mathrm{Gl}$ and G2 are best understood as the giving of reasons for the truth of G3? I rather doubt that this will be the best explanation of why $\mathrm{G}$ was said or written, for it is highly improbable on its face that anyone could think G1 or G2 reasons to believe G3. (I don't even know what it would be like to think this.) Still, if someone thinks $G$ is an argument he might well ask whether G1 and $\mathrm{G} 2$ are dependent or independent. I have no idea how he would answer this question, but he might consider "neither" or "undecidable" or "too problematic" as allowable answers. Perhaps this is one of those philosophical problems that Nietzsche advised us to treat like cold baths and get in and out of quickly. 


\section{Notes}

1 Terminology is not uniform here. What I prefer to call "dependent-independent" reasons both here and in my book, Basic Logic, are named by Thomas "linked-convergent," while Copi prefers to speak of "premises which jointly support the conclusion" as opposed to "independent premises."

2 These are various informal ways I have used to capture the distinction in my Basic Logic (St. Paul: West Publishing Co., 1988), p. 43.

3 Irving M. Copi, Introduction to Logic, 7th ed. (New York: Macmillan, 1986), p. 21.

4 Stephen Thomas, Practical Reasoning in Natural Language, 3rd ed. (Englewood Cliffs, NI: Prentice-Hall, 1986), p. 61. See the appendix in this edition, "On the history and theory of the linked-convergent distinction."

5 Trudy Govier, A Practical Study of Argument, 2nd ed. (Belmont, $\mathrm{CA}$ : Wadsworth Publishing Co., 1988), p. 127.

6 See the appendix to Chapter 2 of Yanal, Basic Logic, pp. 53-55.

7 David Hitchcock, a referee of this paper, worries about a possibility that can be described this way: Suppose we have an argument, $P l$, $P 2$, therefore $C$. Suppose that $\mathrm{pr}(\mathrm{CIPI})=0.3$ and that $\operatorname{pr}(\mathrm{CIP} 2)=0.4$. Suppose summed ordinarily they yield $\mathrm{pr}(\mathrm{ClP} 1+\mathrm{P} 2)=0.58$. But now Hitchcock raises the possibility that $\operatorname{pr}(\mathrm{P} L \mathrm{P} 2)=1.0$. Perhaps this is an example:
"Either she brings all the money or she leaves town; and she brings all the money; so she doesn't leave town." This is, of course, a deductively invalid argument; but my criterion might still suggest that its premises are dependent. Yet the minor premise deductively entails the disjunctive premise ("P, therefore $\mathrm{P}$ $\checkmark Q^{\prime \prime}$ is a truth functionally valid form). Yet I fail to see how this boobytraps my criterion. Statements $S 1$ and $S 2$ can function as premises for a conclusion $\$ 3$ in some arguments. Other arguments can propose $S l$ as a reason to believe $\mathbf{S 2}$. And it seems to me therefore irrelevant to determining the structure of a certain argument that in another argument than the one under consideration "She brings the money" entails "Either she brings the money or she leaves town."

8 The occasion for writing this short piece was a reply to a paper on the topic by Mark Vorobej read at the Chicago meeting of the American Philosophical Association in April 1991. I wish to acknowledge the contributions of referee Tjark Kruiger who cleaned up some terminology, and David Hitchcock who raised the possibility taken up in the previous note as well as the issue addressed through examples $\mathrm{E}, \mathrm{F}$, and $\mathrm{G}$.

\section{INQUIRY

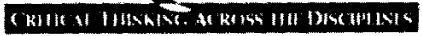

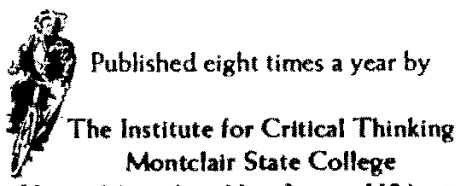 \\ Upper Montclair, New Jersey, USA 07043}

Wendy Oxman-Michelli, Director Mark Weinstein, Associate Director Robert Michael Esformes, Editor

Subscriptions are presently free of charge. For further information, to request a subscription, or for a list of other Institute publications, write us or call (201) 893.5184 . 